\title{
Exploring Fluorescence and Fragmentation of Ions Produced by Electrospray Ionization in Ultrahigh Vacuum
}

\author{
Konstantin Chingin, ${ }^{\mathrm{a}}$ Huanwen Chen, ${ }^{\mathrm{b}}$ Gerardo Gamez, ${ }^{\mathrm{a}}$ \\ and Renato Zenobi ${ }^{\mathrm{a}}$ \\ ${ }^{a}$ Department of Chemistry and Applied Biosciences, ETH Zürich, Zürich, Switzerland \\ ${ }^{\mathrm{b}}$ Applied Chemistry Department, East China Institute of Technology, Fuzhou, China
}

\begin{abstract}
Fluorescence spectroscopy and mass spectrometry have been extensively used for characterization of biomaterials, but usually separately. An instrument combining fluorescence spectroscopy and Fourier-transform ion cyclotron resonance mass spectrometry (FTICR-MS) has been developed to explore both fluorescence and mass spectrometric behavior of ions produced by electrospray ionization (ESI) in ultra high vacuum $\left(<5 \times 10^{-9} \mathrm{mbar}\right)$. Using rhodamine $6 \mathrm{G}(\mathrm{R} 6 \mathrm{G})$ as a sample, the instrument was systematically characterized. Gas-phase fluorescence and mass spectral signal of the same ion population are detected immediately after each other. Effects of gas pressure, ion density, and excitation laser power on the fluorescence signal intensity and mass spectral fragmentation patterns are discussed. Characteristic times of ion photodissociation in ultra high vacuum were recorded for different irradiation powers. Photofragmentation patterns of rhodamine 6G ions in the Penning trap of an FTICR spectrometer obtained by photoinduced dissociation (PID) with visible light and sustained off-resonance irradiation collision-induced dissociation (SORI-CID) were compared. The lowest energy dissociation fragment of rhodamine 6G ions was identified by relating PID patterns of rhodamine $6 \mathrm{G}$ and rhodamine 575 dyes at various irradiation powers. The unique instrument provides a powerful platform for probing the intramolecular relaxation mechanisms of nonsolvated ions when interacting with light, which is of great fundamental interest for better understanding of their physical and chemical properties. (J Am Soc Mass Spectrom 2009, 20, 1731-1738) (c) 2009 American Society for Mass Spectrometry
\end{abstract}

$\mathrm{F}$ luorescence spectroscopy is widely applied for sensitive probing of the structure of matter due to its high specificity to the microenvironment. It can provide several types of information on a large variety of samples, such as solid surfaces, biological membranes, living cells, etc [1]. One powerful feature is that it allows one to obtain structural details of biomolecules. For instance, fluorescence spectroscopy allows determining binding sites of a protein, as well as its conformational transitions or intramolecular distances. Also, this technique yields information on flexibility, helix structure, torsion dynamics, and carcinogenesis of nucleic acids [1]. Typically, labeling with fluorescent probes is required since most biomolecules do not have intrinsic fluorophores. Protein tagging is nowadays routinely achieved using commercially available fluorescent labels that covalently bind to amino or sulfhydryl groups.

Recently, fluorescence spectroscopy has started to be used to probe ions in the gas phase [2-7]. This complements solution phase data and is important for eluci-

Address reprint requests to Dr. R. Zenobi, Chemistry Department and Applied Biosciences, ETH Zürich, CH-8093 Zürich, Switzerland. E-mail: zenobi@org.chem.ethz.ch dating the role of the solvent in stabilizing a native structure. Also, gas-phase data are much easier to relate to theoretical calculations because in such a case it is not necessary to consider the interaction of a molecule with solvent molecules, which greatly simplifies the calculations. Nonvolatile molecules are usually brought into the gas phase in ionic form by soft ionization techniques, such as electrospray ionization (ESI) [8] or matrix-assisted laser desorption/ionization (MALDI) [9]. Ions produced are typically guided into a quadrupolar trap where they can be stored for a long time as a compact cloud, which enables recording of their fluorescence $[3,4,6,7]$. In their pioneering work, Parks and coworkers monitored dissociation of double-stranded oligonucleotide anions in the gas phase by observing the change in the efficiency of fluorescence resonance energy-transfer (FRET, [10]) between a pair of fluorophores BODIPY-TMR (donor) and BODIPY-TR (acceptor) labeling two strands of the duplex [11]. Later, the same group described the gas-phase behavior of a small Trp-cage protein such as its folding/unfolding dynamics [12]. Again, they used BODIPY-TMR dye as a reporter because its fluorescence intensity depends strongly on its proximity to the intrinsic Trp residue of a protein. Quenching of the dye fluorescence was due to 
photoinduced electron-transfer (PET), which depends exponentially on fluorophore-quencher separation [13]. The same methodology was applied to explore conformational changes in unsolvated ions of polyproline peptides and a $\beta$-hairpin peptide [14].

Fourier-transform ion cyclotron resonance mass spectrometry (FTICR-MS) [15] is a powerful technique which has been successfully applied in a variety of rapidly developing research areas of modern biochemistry, such as proteomics and petroleomics. Up to now, fluorescence detection in the Penning trap of an FTICR spectrometer has only been performed in home-built instruments with internal ionization sources, such as electron impact (EI) [16] or internal MALDI [5]. This substantially limits the application range of these experimental setups.

Our previous experiments were performed in an FTICR mass spectrometer with an internal MALDI ionization source described in detail elsewhere [5]. Clear evidence of FRET occurring in the gas phase was demonstrated for the system rhodamine 6G (donor) and sulforhodamine B (acceptor) covalently bound through a phenyl ring linker [17]. One key limitation of this setup was the low molecular weight range $(\mathrm{m} / \mathrm{z}<3000)$, because the relatively fast ions produced by the MALDI internal source could not be efficiently slowed down before entering the ICR cell. For this reason, heavier ions having higher kinetic energy could not be trapped. Also, since no ion optics were used between the MALDI target and the ICR cell, the ion cloud entering the cell was not focused in the radial direction. Therefore, quadrupolar axialization was always necessary to bring the ions onto the $\mathrm{z}$-axis to provide a good overlap with the laser-induced fluorescence (LIF) excitation laser beam. The axialization in turn requires elevated buffer gas pressure ( $>10^{-5}$ mbar). No fluorescence signal was observed without axialization.

Here we present a novel experimental setup for fluorescence and dissociation spectroscopy experiments on gas-phase ions produced in an ICR mass spectrometer using a commercial ESI source. The setup opens new perspectives for spectroscopic probing of biomolecular ions under conventional FTICR-MS experimental conditions. If operated at ultra-high vacuum pressures, the FTICR offers superb mass spectral performance, including high mass accuracy, highresolution, and $\mathrm{MS}^{n}$ capabilities for the identification of ions using accurate mass measurements and by observing fragmentation processes through radical or neutral losses. The setup described here offers high versatility as the fluorescence collection is essentially decoupled from the ion optics between the ESI source and the Penning trap. Therefore, our setup can be easily installed on other commercial FTICR instruments, no modifications being required except the installation of the measuring cell described in detail in the Experimental section.

\section{Experimental}

\section{Setup}

The experiments described here were performed on an FTICR mass spectrometer with an electrospray ionization (ESI) source (IonSpec Corporation, Lake Forest, CA, USA) and a 4.7 Tesla superconducting magnet (Bruker, Fällanden, Switzerland). A completely new fluorescence setup, including a new ICR cell, was engineered in our laboratory because the previous setup was not compatible with the z-axis ion introduction by external ESI [5].

Ions produced by ESI at atmospheric pressure were guided through a hexapole transport system with differential pumping into an open cylindrical cell. The diameter of the cell is $5 \mathrm{~cm}$. All the electrodes were machined from OFHC copper (Haeuselmann Metal AG, Dietlikon, Switzerland). Holes of $6 \mathrm{~mm}$ in diameter were drilled into the excitation and detection plates to allow for a laser beam to be introduced into the cell.

The latest version of our experimental setup for the LIF measurements is shown schematically in Figure 1a. The trapped ions were irradiated by a CW Ar ion laser (Innova 300; Coherent, Santa Clara, USA) at a wavelength of $488 \mathrm{~nm}$. The laser beam was introduced into the vacuum chamber through a high vacuum glass viewport (PF610010-X; Pfeiffer Vacuum, Asslar, Germany). Due to space limitations caused by the magnet core size (Figure 1), the beam was introduced into the ICR cell through a right angle reflector (74-90-UV; OceanOptics, Dunedin, FL, USA). It consists of a mirror mounted on an anodized aluminum holder so that the incident light is reflected at a $90^{\circ}$ angle into the cell. To efficiently absorb the laser beam after it has crossed the ion cloud, a conical beam dump (PL15; Newport, Irvine, CA, USA) was mounted on the opposite side. Light would have to reflect at least three times from black surfaces (reflectivity of less than $10^{-4}$ ) to escape, but most of it undergoes many more. Note that we cannot use a "woods horn" beam dump because its installation would require much more free space in the radial direction of the vacuum chamber than available. To suppress scattered light further, two home-built baffle systems were introduced as shown in Figure 1. Finally, the inner walls of the FTICR cell were coated with vacuum compatible, electrically conductive paint (Aquadag E; Acheson Co., Port Huron, MI, USA), which dramatically reduced the scattered excitation laser light.

Alignment of the laser beam path was done when the cell was outside of the vacuum chamber. A long baffle was rigidly braced to the viewport on the flange. Two slits, $2 \mathrm{~mm}$ in diameter, were mounted inside the baffle. The distance between the slits was $50 \mathrm{~cm}$. These slits were then used to guide the laser beam when the cell was inside the vacuum chamber. Figure $1 b$ shows a photograph of the ICR cell during the alignment procedure. The conical beam dump was removed to control the position of the beam in the cell. 

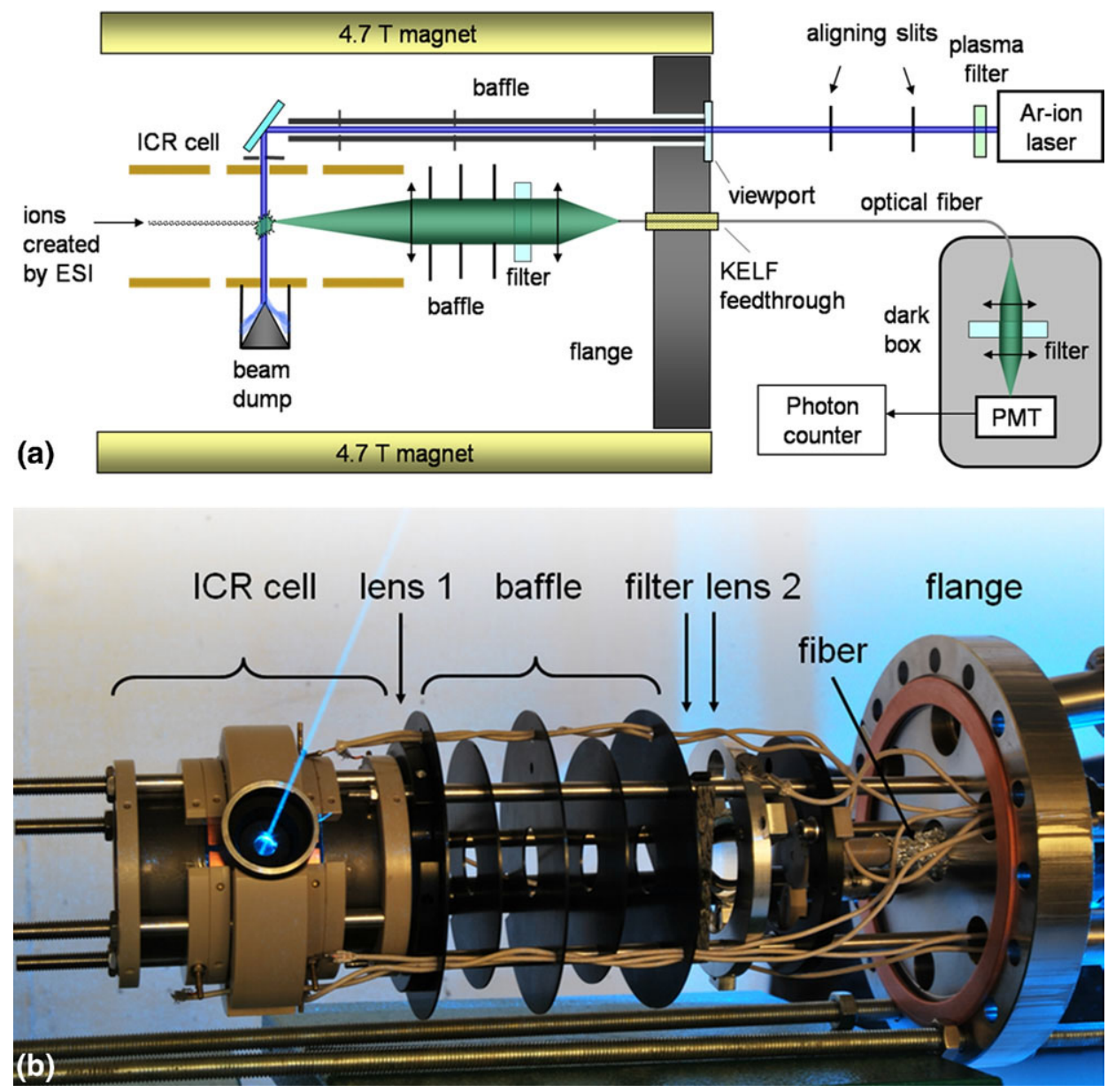

Figure 1. (a) Experimental setup. (b) ICR cell during the laser beam alignment procedure. The conical beam dump was removed to show the position of the beam inside the cell.

The ion cloud fluorescence was collected by a system of lenses (lens 1: ACH 25X50 MGF2TS, Edmund, $\mathrm{f}=50$ $\mathrm{mm}$; lens 2: 01LAG117/066, Melles Griot, Rochester, NY, USA; $\mathrm{f}=25 \mathrm{~mm}$ ), and focused onto the cleaved edge of a $3 \mathrm{~mm}$ core diameter plastic fiber with a high NA value of 0.51 (PGR FB 3000, SEDI, Courcouronnes, France). A long pass filter (LP02-488RU; RazorEdge, Semrock, Rochester, NY, USA) was used after the collimating lens to block a part of the scattered laser light. The distance between the collimating and the focusing lens was $10 \mathrm{~cm}$.

The fiber passes the vacuum flange through a Kel-F feedthrough [18]. Light exiting the fiber was focused onto the active surface of a photomultiplier tube (R446; Hamamatsu, Hamamatsu City, Japan). A long pass filter (FEL0500; Thorlabs, North Newton, NJ, USA) was used to block the scattered excitation laser light that was not blocked in the vacuum chamber. The signal from the photomultiplier tube was recorded by an $800 \mathrm{MHz}$ gated photon counter/multiscaler (PMS 400; Becker and Hickl $\mathrm{GmbH}$, Berlin, Germany).

Visible spectra were collected by coupling the plastic fiber to a spectrograph (Shamrock SR-163; Andor, Northern Ireland) with a $100 \mu \mathrm{m}$ entrance slit and a CCD camera (DV420A-OE, iDus; Andor, Northern Ireland).
The sequence of events in a typical experiment started from creating ions by ESI. These ions were then guided through a system of hexapole filters into the ICR cell. When an increase of the buffer gas pressure was necessary, a pulse of helium gas was introduced through a pulsed leak valve. Ions were stored in the cell as long as necessary (from $1 \mathrm{~s}$ to $5 \mathrm{~min}$ ). Cyclotron motion of the trapped ions was then excited to record a mass spectrum. The Ar ion laser and the PMT were running constantly during the entire experiment. Background signal was therefore detected continuously (between 350 and 1500 counts per s, depending on the excitation laser power). When the ions entered the cell, the signal increased due to their fluorescence (up to 10 000 counts per s for R6G ions). Excitation of the cyclotron motion broke the spatial overlap of the axialized ion cloud and the Ar ion laser beam, i.e., fluorescence was no longer recorded after that event. Note that the recorded mass spectrum shows all the photo-induced fragments produced during the ion cloud storage in the cell. Supplemental material, Figure S1, which can be found in the electronic version of this article, summarizes time profile of the major events during a single experiment. 
For the CID experiments, sustained off-resonance irradiation (SORI) was applied with argon as a collision gas. The peak pressure was $4 \times 10^{-6} \mathrm{mbar}$, the offset frequency $800 \mathrm{~Hz}$, irradiation duration $300 \mathrm{~ms}$, and the amplitude ranged from 0.5 to $2.5 \mathrm{~V}$. Some design features aimed at further improving the analytical performance of the platform are discussed in Supplementary material.

\section{Materials}

Rhodamine 6G (R6G) was purchased from Acros Organics, Liege Area, Belgium. Rhodamine 575 (R575) was purchased from Exciton, Dayton, OH, USA. Work solutions $(0.5 \mu \mathrm{M})$ were prepared in an water/acetonitrile (3:7 vol:vol) mixture with $1 \%$ acetic acid added.

\section{Results and Discussion}

\section{Ion Fluorescence in Ultra High Vacuum}

It is worth noting that the light absorption/emission of polar dyes can significantly change when going from solution to the gas phase. Specifically, gas-phase R6G ions absorb the $488 \mathrm{~nm}$ laser line much better than the other major line of the Ar ion laser at $514 \mathrm{~nm} \mathrm{[17],}$ suggesting that the absorption maximum lies close to $488 \mathrm{~nm}$. In contrast, absorption of R6G in ethanol peaks at around $530 \mathrm{~nm}$. A considerable blue shift of $\approx 40 \mathrm{~nm}$ of the R6G absorption can therefore be estimated to occur when moving from solution to the gas phase. This illustrates how sensitive fluorescence spectroscopy is to changes in the microenvironment.

Figure 2 shows fluorescence recorded from R6G ions irradiated at $488 \mathrm{~nm}$ at $0.2 \mathrm{~mW} / \mathrm{mm}^{2}$. This low irradiance was used to make sure no photodissociation took place during the experiment. This was confirmed by the mass spectrum, which showed only molecular ions of R6G (not shown). It can be seen that it takes some time

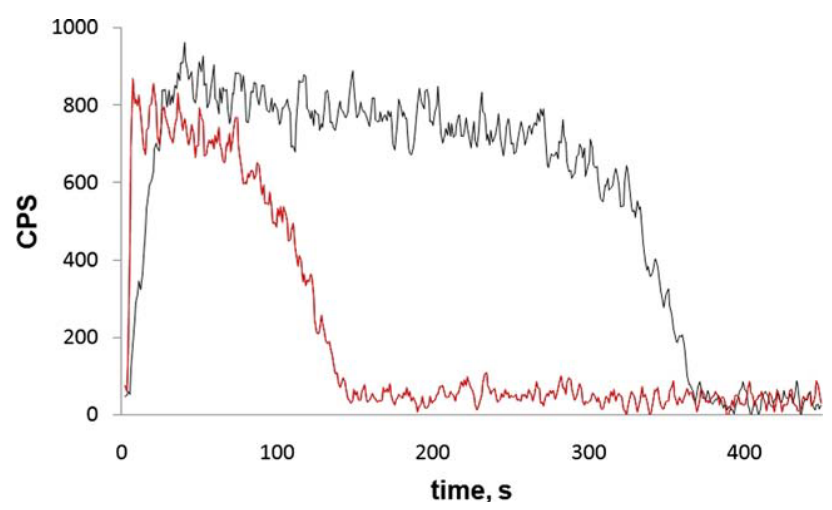

Figure 2. Two fluorescence signals recorded from rhodamine 6G ions irradiated at $488 \mathrm{~nm}$ with an irradiance of $0.2 \mathrm{~mW} / \mathrm{mm}^{2}$. Increase in the signals started right after the ions had entered the cell. Black curve: no neutral gas was introduced during the ion cloud storage in the ICR cell. Red curve: eight $10 \mathrm{~ms}$ helium gas pulses with a $200 \mathrm{~ms}$ interval were introduced right after the ions had entered the cell. The signals were background subtracted. after the ions enter the cell before the signal reaches a plateau (black curve). When eight $10 \mathrm{~ms}$ pulses of helium gas with a $200 \mathrm{~ms}$ interval were introduced into the cell right after the ion injection, the peak pressure being $5 \times 10^{-5}$ mbar, the signal reached its maximum much faster (red curve). We believe that this results from more efficient axial cooling of the ion cloud along the magnet field lines. At elevated pressure, a much shorter time is needed to focus ions axially in the ICR cell, which makes for a faster complete overlap of the ion cloud with the laser beam [16]. In case of lower pressure, a much longer time is required to cool the ion cloud and, therefore, complete overlap is achieved much later. The cooling can be indirectly observed by monitoring the intensity of the MS signal at different times. For short ion storage periods when the ion cloud in the cell is not completely focused, MS signal intensity is lower compared with that after the ions have been equilibrated. This is because the excitation field is stronger in the center of the ICR cell and, therefore, a better focused ion cloud would produce a stronger signal [19]. Also, the fact that the fluorescence signal grows while the ion cloud is getting more compact further indicates that the laser beam does go through the center of the cell. Otherwise, if the interaction with the ion cloud would take place at the periphery, the signal would decay while the ions were being focused towards the center of the cell.

Another interesting observation is that the fluorescence signal decays whether or not gas was introduced, although no ion photodissociation occurred. We believe that the reason for this observation is the magnetron expansion of the ion cloud inside the ICR cell [15], which causes a gradual deterioration of the overlap between the laser beam and the ion cloud, resulting in the signal decay. Also, the MS signal intensity starts to decrease after some time as a result of the ion losses in the cell. The fluorescence signal decay was faster when helium gas was introduced (Figure 2, red curve), which enhances the magnetron expansion as its rate strongly depends on the background pressure [15].

Quadrupolar axialization is often performed to avoid magnetron expansion of the ion cloud in the ICR cell [15]. This, however, requires elevated pressure in the trap and therefore was not applied in our experiments.

Figure S3 (Supplementary material) shows a correlation of the fluorescence signal with the intensity of a molecular peak for R6G ions in the mass spectrum, in the absence of photodissociation. In ICR the latter depends linearly on the number of ions in the cell. One can see that the dependence in the figure deviates from linearity at higher MS signal intensities. This can be explained by incomplete overlap between the ion cloud and the laser beam, since the ion cloud expands at high ion densities due to space charge effects. Better overlap can be achieved using a laser beam of a bigger diameter. This would, however, result in more pronounced laser light scattering inside the cell. For this reason, no attempt was undertaken to improve the overlap in this 
fashion. For moderate ion densities, which are typically used for optimal ICR-MS performance, the fluorescence signal does not deviate from linearity significantly (Figure S3). This implies overlap of $\sim 100 \%$ between the laser cross-section and the ion cloud in this case. The reproducibility of fluorescence intensity measurements increased with an increasing number of ions in the cell. For normal ICR-MS operating conditions, the scatter in fluorescence signal did not exceed 10\% comparing different experiments with ion populations producing equal MS signals.

\section{Effects of Gas Pressure and Laser Power on the Fluorescence Signal}

In earlier studies, it was demonstrated that the fluorescence signal from gas-phase ions depends strongly on the buffer gas pressure $[4,6,7,17]$. Buffer gas molecules vibrationally cool excited ions, preventing them from rapid photodissociation. The higher the pressure, the more efficient the cooling, which allows using higher excitation laser power. Figure S4 (Supplementary material) demonstrates the dependence of the highest achievable fluorescence signal recorded from R6G ions in an ICR cell on buffer helium gas pressure recorded by Dashtiev et al. [17]. The attainable signal grew as the pressure increased, due to the possibility to use higher laser powers without destroying fluorescent ions. Quadrupolar axialization was applied to keep the ions in the center of the cell while the fluorescence was recorded. No photodissociation occurred at laser irradiances up to $50-65 \mathrm{~mW} / \mathrm{mm}^{2}$ at a background gas pressure around $10^{-4}-10^{-5} \mathrm{mbar}$, at least on the time scale of $10 \mathrm{~s}$. Also, the fluorescence yield was found to

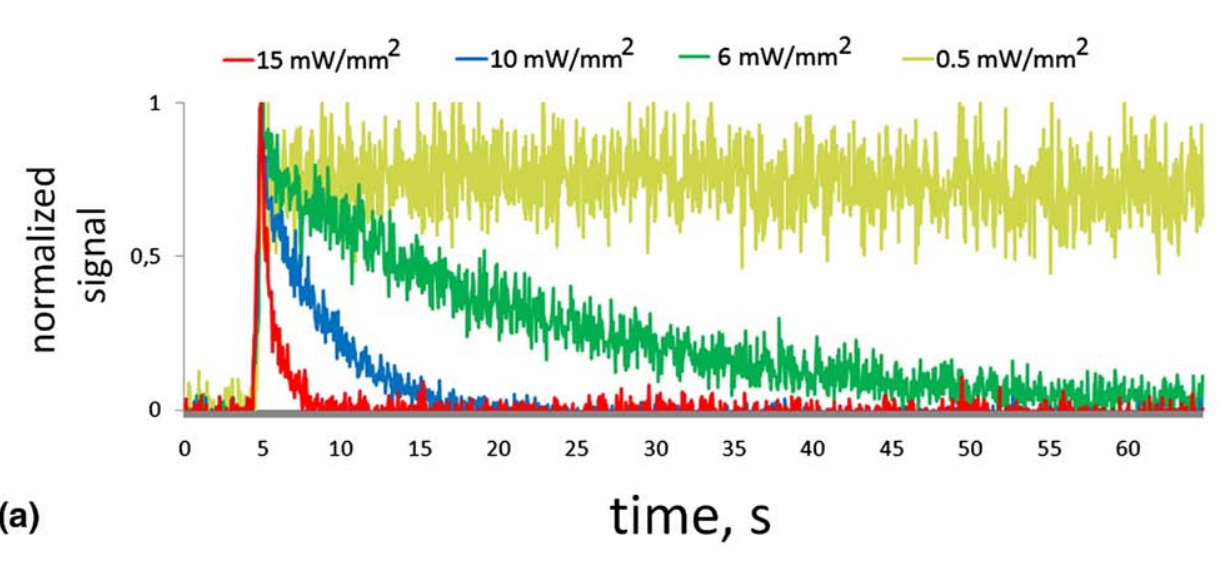

(a)

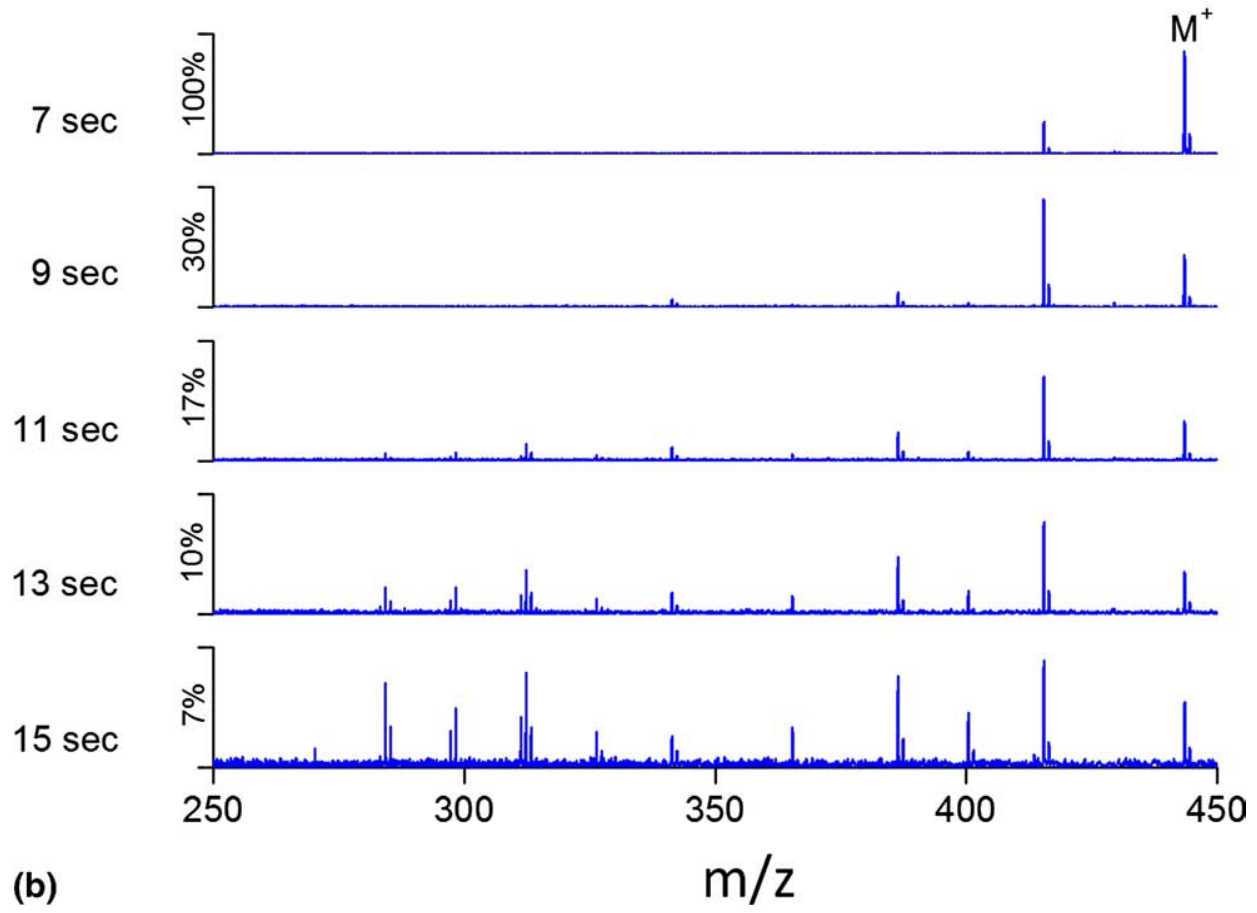

Figure 3. (a) Fluorescence signal decay due to photodissociation of rhodamine 6G ions at different excitation irradiances. (b) Evolution of the MS spectrum for rhodamine 6G ions due to photodecomposition. Laser irradiance was $10 \mathrm{~mW} / \mathrm{mm}^{2}$ (blue curve in Figure 3a). All spectra are normalized to the signal intensity of its corresponding largest peak. The y-axis shows the relative intensity of each spectrum with respect to the $\mathrm{M}^{+}$signal of the spectra at $7 \mathrm{~s}$. 
depend on what kind of buffer gas was used for the quadrupolar axialization [20]. In particular, no fluorescence from R6G ions could be detected when argon gas was applied, which was explained by possible quenching due to the external heavy atom effect [1]. The highest fluorescence yield was obtained by helium gas as a buffer.

Clearly, in ultrahigh vacuum where collisions with neutrals are very rare, vibrational heating of ions should proceed very rapidly. Figure $3 a$ shows the fluorescence signal from R6G ions recorded in our new setup at various excitation laser irradiances. Five time profiles were accumulated for each laser irradiance. The background signal was subtracted, and the resulting signals were then normalized. The rapid increase of the signal at around $4 \mathrm{~s}$ corresponds to the time when the ions enter the ICR cell. A short helium gas pulse was introduced as soon as they had been trapped to accelerate axial focusing of the ion cloud. The decay is due to photodissociation of parent R6G ions into nonfluorescing fragments (Figure $3 b$ ). It is worth noting that at least the first fragment of R6G at $m / z 415$, produced by loss of the ethylene, was found to fluoresce as well, which could be seen by illuminating the isolated ion species at $m / z 415$ (not shown). The structural and photochemical properties of the first fragment will be discussed in detail below. The decay curves thus represent the net fluorescence profile from both R6G ions and their fragments. These curves were well fit with a double exponential $[\mathrm{y}=\mathrm{A} 1 \exp (-\mathrm{t} / \mathrm{a} 1)+\mathrm{A} 2 \exp (-\mathrm{t} / \mathrm{a} 2)+\mathrm{y} 0$, $\mathrm{A} 1=431, \mathrm{a} 1=3 \mathrm{~s}, \mathrm{~A} 2=1219$, a2 $=89 \mathrm{~s}, \mathrm{R}^{2}=0.97$ for the decay at $10 \mathrm{~mW} / \mathrm{mm}^{2}$ in Figure $3 a$ (blue curve)], which suggests that the role of the fragments in producing the net signal cannot be neglected. We interpret the second, slower time constant to be due to the fluorescence from fragments that exhibit quite a different branching ratio between the different excited-state decay channels (fluorescence, nonradiative decay, photodissociation), presumably due to the structural changes. Figure $3 \mathrm{~b}$ shows how the mass spectrum evolves due to photofragmentation of R6G. The spectra correspond to the blue decay curve in Figure 3a, providing a complete time profile of photochemical dissociation reactions that take place in the ICR cell.

Indeed, rapid photodissociation limits the fluorescence detection efficiency. It can be seen from Figure 3a that at a laser irradiance of $15 \mathrm{~mW} / \mathrm{mm}^{2}$, the fluorescence signal decays completely within about $5 \mathrm{~s}$ after the ions have entered the cell. However, as mentioned earlier, at elevated pressures no photodissociation of R6G ions in an ICR cell could be seen at a laser irradiance as high as $65 \mathrm{~mW} / \mathrm{mm}^{2}$, at least on a time scale of $10 \mathrm{~s}$ [17]. For comparison, the probability of R6G photobleaching in solution, where vibrational cooling is much more efficient than in gas phase due to frequent collisions with solvent molecules, is in the order of $10^{-6}-10^{-7}$ for irradiances below $10 \mathrm{~W} / \mathrm{mm}^{2}$, which results in fluorescence life times about $1000 \mathrm{~s}$ [21].

\section{Photoactivation in Ultra High Vacuum}

To obtain information on the structure of biomolecular ions in FTICR mass spectrometry, sustained offresonance irradiation collision-induced dissociation (SORI-CID) is often used [22]. However, it only allows for activation of ions with a certain $\mathrm{m} / \mathrm{z}$ value and, therefore, prevents further dissociation of produced fragments. For large biomolecules, it can significantly limit attainable structural information on a parent ion [23]. Also, since SORI-CID is performed at an elevated pressure, the fragments produced may diffuse radially due to magnetron expansion promoted by the gas load. This can significantly compromise the accuracy of the mass measurement [24]. In case of photo-induced activation, however, no gas introduction is necessary, and daughter ions are still available for further fragmentation as long as they are spectroscopically active. Here we show that even for small ions, a difference exists between SORI-CID and photoinduced dissociation (PID) MS spectra. Figure 4 demonstrates SORI-CID and PID spectra for R6G ions obtained at different activation energies. It can be seen that at low CID energy, ions at

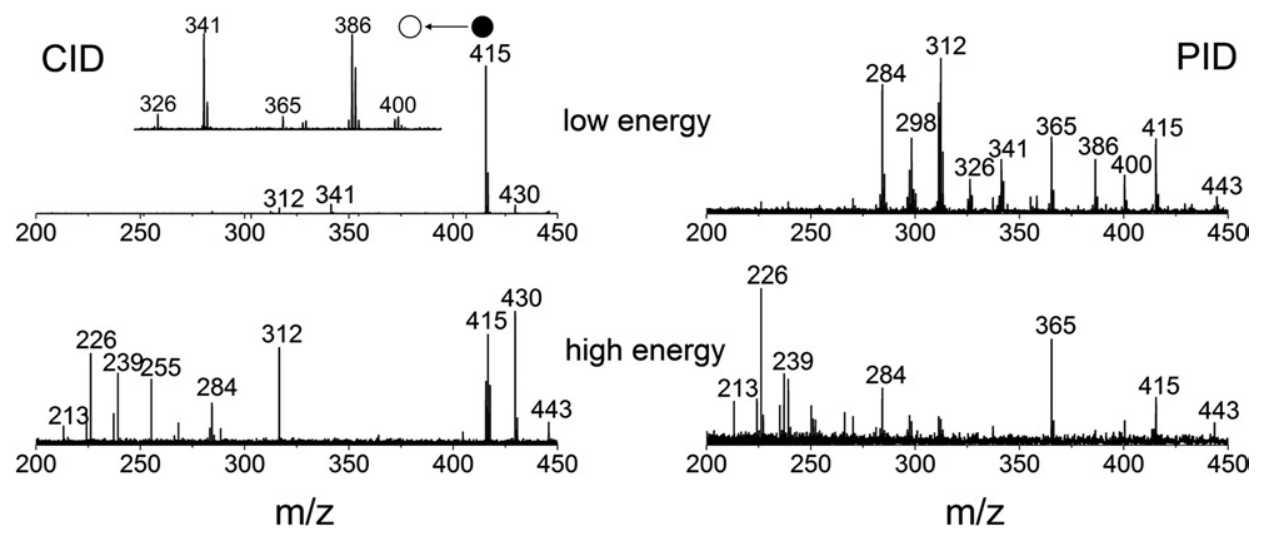

Figure 4. SORI-CID (left) and PID (right) MS spectra obtained at different activation energies (SORI-CID voltages were 1.2 and $1.5 \mathrm{~V}$, laser irradiance was $10 \mathrm{~mW} / \mathrm{mm}^{2}$ and $20 \mathrm{~mW} / \mathrm{mm}^{2}$ for the top and bottom spectra respectively). Inset: SORI-CID MS spectrum of the fragment at $\mathrm{m} / \mathrm{z} 415$. 


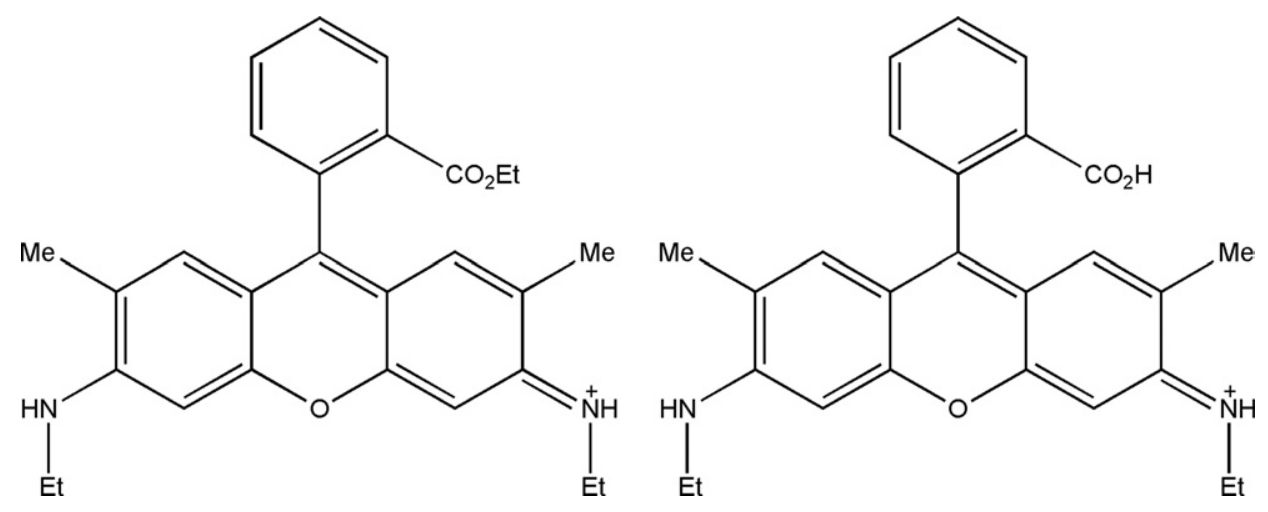

Scheme 1. Structures of rhodamine 6G (left) and rhodamine 575 (right) ions.

$\mathrm{m} / \mathrm{z} 415$ are preferentially produced. Once produced, these ions are not available for further fragmentation in SORI-CID. Low-energy PID gives the same fragment at $\mathrm{m} / \mathrm{z}$ 415. It was observed, however, that despite some structural changes, this fragment also absorbs light at the same wavelength as the parent R6G ions. Therefore, it can dissociate further, resulting in a more complete fragmentation pattern as can be seen by comparing top left and top right spectra in Figure 4 . For example, the fragments at $\mathrm{m} / \mathrm{z} 386$ and 365 are absent in the SORICID spectra, while being easily detected by PID. The fragmentation patterns suggest that these species are much easier to produce from the fragment at $\mathrm{m} / \mathrm{z} 415$ rather than directly from the parent R6G ions. To prove this hypothesis, SORI-CID was performed on the fragment at $\mathrm{m} / \mathrm{z} 415$, revealing abundant signals at $\mathrm{m} / \mathrm{z} 386$ and 365 (inset in Figure 4). At higher activation energies, the difference between CID and PID patterns is not so pronounced because the fragment at $\mathrm{m} / \mathrm{z} 415$ is no longer the main dissociation channel (Figure 4, bottom).

It is worth noting that excitation of fluorescent molecules with visible light in high vacuum results in gradual gain of internal energy during successive photon absorption/emission cycles. Photofragmentation happens when accumulated vibrational energy is high enough to break the weakest chemical bond. This is very similar to the mechanism of infrared multiphoton dissociation (IRMPD) [23] where vibrational energy is accumulated directly through successive absorption of IR quants, without exciting the higher electronic energy level.

For molecules that absorb visible light but do not fluoresce (quenchers), excitation with visible light will, however, result in a much faster accumulation of vibrational energy due to internal conversion from the excited electronic state and therefore very rapid fragmentation. If dissociation proceeds faster than complete vibrational energy redistribution (IVR), some complementary structural information can be accessed (see the discussion in Supplementary material). Alternatively, excitation to the second excited state can be achieved for higher laser irradiances, in which case photophysics is essentially the same as for UV excitation [25].
The lowest activation energy at which fragmentation starts to occur is characteristic of R6G photostability. It is thus important to know how the first fragment at $\mathrm{m} / \mathrm{z}$ 415 is created. The structure of an R6G ion is shown in Scheme 1 (left). The difference of 28 units is obviously due to the loss of an ethylene. It is not obvious, however, which $\mathrm{C}_{2} \mathrm{H}_{4}$ group dissociates, among the three available. We believe that most probably it is the one from the ester group. If this is the case, the resulting fragment should look like in Scheme 1 (right), the structure of rhodamine 575 (R575), which is commercially available on the market. To prove our hypothesis, PID spectra of these two compounds were obtained at different excitation laser powers (Figure 5). A remarkable agreement between these two sets of data could be seen. This finding suggests that with high probability the lowest energy fragmentation channel of R6G ions is dissociating into R575 ions due to loss of ethylene from the ester. Also, R575 was found to be fluorescent when activated at $488 \mathrm{~nm}$, which is consistent with our finding that some
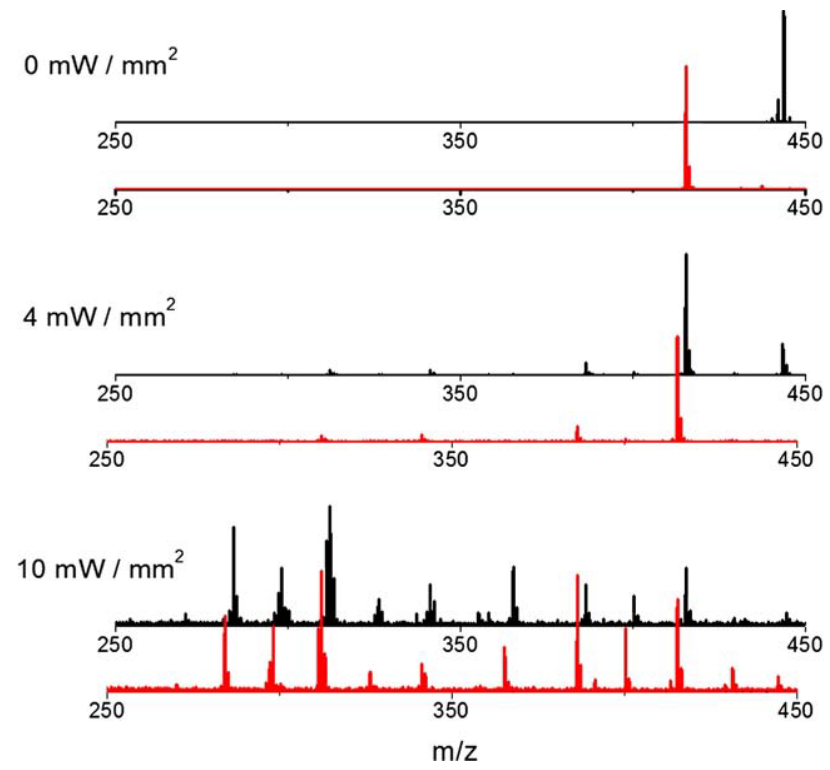

Figure 5. PID spectra of rhodamine 6G (black line) and rhodamine 575 (red line) ions at different excitation laser powers. 
fragments of R6G contribute into the net signal, as discussed above. The fluorescence yield of R575 was about two times lower than that of R6G, which is probably due to the shift in absorption/emission maxima.

\section{Conclusions}

In combination with fluorescence/photofragmentation spectroscopy FTICR-MS provides unique capabilities for probing ions in the gas phase due to the long ion storage times, ultra low-pressure, and its high mass selectivity. A unique setup was built in our lab for this purpose, allowing us to perform fluorescence spectroscopy inside an FTICR spectrometer on ions produced by electrospray ionization. The new platform was thoroughly described, and preliminary data on gaseous rhodamine $6 \mathrm{G}$ ions were shown.

\section{Acknowledgments}

The authors thank Mr. Kurt Baumgartner for machining the parts of the ICR measuring cell and Mr. Heinz Benz for the technical assistance with electronics. The authors also thank Dr. Arno Wortmann (Roche, Switzerland), Dr. Maxim Dashtiev (Bruker, Russia), Dr. Vladimir Azov (University of Bremen, Germany), and Dr. Michael Gorshkov (Institute of Energy Problems of Chemical Physics, Russia) for helpful discussions.

\section{Appendix A Supplementary Material}

Supplementary material associated with this article may be found in the online version at doi:10.1016/ j.jasms.2009.05.011.

\section{References}

1. Valeur, B. Molecular Fluorescence: Principles and Applications; Wiley-VCH: Weinheim, 2002, 11-15, 41.

2. Wang, Y.; Hendrickson, C. L.; Marshall, A.G. Direct Optical Spectroscopy of Gas-Phase Molecular Ions Trapped and Mass-Selected by Ion Cyclotron Resonance: Laser-Induced Fluorescence Excitation Spectrum of Hexafluorobenzene (C6F6+). Chem. Phys. Lett. 2001, 1/3, 69-75.

3. Khoury, J. T.; Rodriguez-Cruz, S. E.; Parks, J. H. Pulsed Fluorescence Measurements of Trapped Molecular Ions with Zero Background Detection. J. Am. Soc. Mass Spectrom. 2002, 6, 696-708.

4. Wright, K. C.; Blades, M. W. Fluorescence Emission Spectroscopy of Trapped Molecular Ions. Proceedings of the 51st ASMS Conference on Mass Spectrometry and Allied Topics; Montreal, Canada, June, 2003.

5. Frankevich, V.; Guan, X. W.; Dashtiev, M.; Zenobi, R. Laser-Induced Fluorescence of Trapped Gas-Phase Molecular Ions Generated by
Internal-Source Matrix-Assisted Laser Desorption/Ionization in a Fourier Transform Ion Cyclotron Resonance Mass Spectrometer. Eur. J. Mass Spectrom. 2005, 5, 475-482.

6. Bian, Q.; Talbot, F. O.; Forbes, M. W.; Yao, H.; Jockusch, R. A. Development and Characterization of Laser Induced Fluorescence Spectroscopy Coupled with Ion Trap Mass Spectrometry. Proceedings of the 56th ASMS Conference on Mass Spectrometry and Allied Topics; Denver, CO, June, 2008.

7. Sassin, N. A.; Everhart, S. C.; Dangi, B. B.; Ervin, K. M.; Cline, J. I. Fluorescence and Photodissociation of rhodamine 575 Cations in a Quadrupole Ion Trap. J. Am. Soc. Mass Spectrom. 2009, 1, 96-104.

8. Fenn, J. B.; Mann, M.; Meng, C. K.; Wong, S. F.; Whitehouse, C. M. Electrospray Ionization for Mass-Spectrometry of Large Biomolecules. Science 1989, 4926, 64-71.

9. Hillenkamp, F.; Karas, M.; Beavis, R. C.; Chait, B. T. Matrix-Assisted Laser Desorption Ionization Mass-Spectrometry of Biopolymers. Anal. Chem. 1991, 24, A1193-A1202.

10. Stryer, L.; Haugland, R. P. Energy Transfer-A Spectroscopic Ruler. Proc. Natl. Acad. Sci. U.S.A. 1967, 2, 719-726.

11. Danell, A. S.; Parks, J. H. FRET Measurements of Trapped Oligonucleotide Duplexes. Int. J. Mass Spectrom. 2003, 1/2, 35-45.

12. Iavarone, A. T.; Parks, J. H. Conformational Change in Unsolvated Trp-Cage Protein Probed by Fluorescence. J. Am. Chem. Soc. 2005, 24 8606-8607.

13. Neuweiler, H.; Sauer, M. Using Photoinduced Charge Transfer Reactions to Study Conformational Dynamics of Biopolymers at the SingleMolecule Level. Curr. Pharm. Biotechnol. 2004, 3, 285-298.

14. Iavarone, A. T.; Duft, D.; Parks, J. H. Shedding Light on Biomolecule Conformational Dynamics Using Fluorescence Measurements of Trapped Ions J. Phys. Chem. A 2006, 47, 12714-12727.

15. Marshall, A. G.; Hendrickson, C. L.; Jackson, G. S. Fourier Transform Ion Cyclotron Resonance Mass Spectrometry: A primer. Mass Spectrom. Rev. 1998, 1, 1-35.

16. Li, G. Z.; Vining, VA.; Guan, S. H.; Marshall, A. G. Laser-Induced Fluorescence of $\mathrm{Ba}+$ Ions Trapped and Mass-Selected in a Fourier Transform Ion Cyclotron Resonance Mass Spectrometer. Rapid Commun. Mass Spectrom. 1996, 14, 1850-1854.

17. Dashtiev, M.; Azov, V.; Frankevich, V.; Scharfenberg, L.; Zenobi, R. Clear Evidence of Fluorescence Resonance Energy Transfer in GasPhase Ions. J. Am. Soc. Mass Spectrom. 2005, 9, 1481-1487.

18. Abraham, E. R. I.; Cornell, E. A. Teflon Feedthrough for Coupling Optical Fibers into Ultrahigh Vacuum Systems. Appl. Optics 1998, 10, 1762-1763.

19. Guan, S. H.; Marshall, A. G. Ion Traps for Fourier-Transform IonCyclotron Resonance Mass-Spectrometry-Principles and Design of Geometric and Electric Configurations. Int. J. Mass Spectrom. Ion Processes 1995, 146, 261-296.

20. Dashtiev, M.; Zenobi, R. Effect of Buffer Gas on the Fluorescence Yield of Trapped Gas-Phase Ions. J. Am. Soc. Mass Spectrom. 2006, 6, 855-858.

21. Eggeling, C.; Widengren, J.; Rigler, R.; Seidel, C. A. M. Photobleaching of Fluorescent Dyes Under Conditions Used for Single-Molecule Detection: Evidence of Two-Step Photolysis. Anal. Chem. 1998, 13, 2651-2659.

22. Gauthier, J. W.; Trautman, T. R.; Jacobson, D. B. Sustained OffResonance Irradiation for Collision-Activated Dissociation Involving Fourier-Transform Mass-Spectrometry-Collision-Activated Dissociation Technique That Emulates Infrared Multiphoton Dissociation. Anal. Chim. Acta 1991, 1, 211-225.

23. Little, D. P.; Speir, J. P.; Senko, M. W.; O'Connor, P. B.; McLafferty, F. W Infrared Multiphoton Dissociation of Large Multiply-Charged Ions for Biomolecule Sequencing. Anal. Chem. 1994, 18, 2809-2815.

24. Guan, S. H.; Marshall, A. G.; Wahl, M. C. MS/MS with High Detection Efficiency and Mass Resolving Power for Product Ions in FourierTransform Ion-Cyclotron Resonance Mass-Spectrometry. Anal. Chem. 1994, 8, 1363-1367.

25. Williams, E. R.; Furlong, J. J. P.; McLafferty, F. W. Efficiency of Collisionally-Activated Dissociation and 193-nm Photodissociation of Peptide Ions in Fourier-Transform Mass-Spectrometry. J. Am. Soc. Mass Spectrom. 1990, 4, 288-294. 